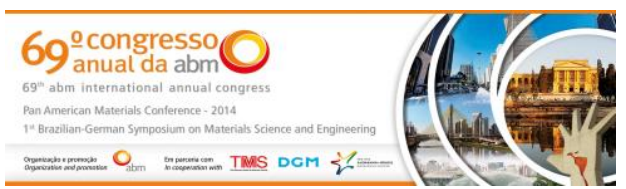

Tema: Gestão de Manutenção

\title{
APLICAÇÃO DE ENGENHARIA DE CONFIABILIDADE NA REDUÇÃ̃O DE PARADAS POR FALHA DA MÁQUINA DE MARCAR DO LAMINADOR DE TIRAS A QUENTE DA ARCELORMITTAL TUBARÃO*
}

Ricardo Tadeu Meneses Sodré1 Laudelino Cota Fonseca Junior ${ }^{2}$ Luiz Rogério Piancar

\section{Resumo}

Quintino Ribeiro Sobrinho ${ }^{4}$

Laminadores de Tiras a Quente (LTQ) são equipamentos que realizam a transformação de placas de aço em bobinas laminadas de aço. Após a fabricação da bobina é necessário à marcação de todas as bobinas laminadas para possibilitar a identificação e rastreabilidade da bobina. A Arcelormittal Tubarão possui uma máquina de marcar automática robotizada que realiza essa identificação. Ocorrendo falha na máquina de marcar a identificação das bobinas passam a ser realizadas manualmente pelo operador, que não possui a mesma velocidade da marcação robotizada, ou seja, imediatamente ocorre perda de produção e após alguns minutos o gargalo se torna insustentável causando a parada do Laminador de Tiras a Quente. Um estudo minucioso foi desenvolvido através da elaboração de um diagrama de blocos funcionais (DBF), levantamento do histórico de falhas e tempos de manutenção, criação das curvas de confiabilidade e mantenabilidade e dos blocos de confiabilidade (RBD) para realização de simulações. Este estudo permitiu direcionar os esforços para os modos de falhas que mais impactavam na produção, reduzindo significativamente as falhas da máquina de marcar. Para sustentabilidade do resultado os planos de manutenção e inspeção foram revisados utilizando a Manutenção Centrada em Confiabilidade (RCM). O estudo proporcionou um aumento de produção de 15.207 ton/ano.

Palavras-chave: Confiabilidade; Máquina Marcar; RCM.

\section{RELIABILITY ENGINEERING APPLICATION REDUCING DOWNTIME BY MARKING MACHINE FAILURES IN THE ARCELORMITTAL TUBARÃO HOT STRIP MILL \\ Abstract}

Hot Strip Mills are equipments that transform slabs in coils. After manufactured the coils, are necessary to provide identification and traceability. The ArcelorMittal Tubarão has an automatic and robotic Marking Machine to realize this identification. If occurs any trouble, the coils identification shall be performed manually by the operator, that not has the same skill and machine velocity, then it immediately causes production losses and after few minutes the bottleneck becomes unsustainable causing Hot Strip Mill Stoppages. A detailed study was developed through the functional block diagram creation; maintenance failures history survey; reliability and maintainability curves creation and the reliability block diagram focusing on realize simulations. This study allowed putting efforts in the failures modes that produced most production impacted, with significantly reducing in the marking machine failures. To guarantee the results sustainability the maintenance and inspection plans have been revised using the Reliability Centered Maintenance (RCM). It gave us a production increase of 15,207 ton/year.

Keywords: Reliability; Marking Machine, RCM.

1 Engenheiro Mecânico, Especialista em Engenharia de Manutenção e em Engenharia de Materiais, Mestre em Fadiga e Mecânica de Fratura, Engenheiro especialista em Manutenção e Gestão de Ativos, Gerência de Tecnologia de Manutenção, ArcelorMittal Tubarão, Vitória, ES, Brasil.

2 Engenheiro Eletricista, Engenheiro especialista em Manutenção e Gestão de Ativos, Gerência de Tecnologia de Manutenção, ArcelorMittal Tubarão, Vitória, ES, Brasil.

3 Engenheiro Mecânico, Engenheiro especialista em Manutenção do Laminador de Tiras a Quente, Gerência de Manutenção do LTQ, ArcelorMittal Tubarão, Vitória, ES, Brasil.

4 Engenheiro Mecânico, Especialista em Engenharia de Manutenção, Engenheiro especialista em Manutenção do Laminador de Tiras a Quente, Gerência de Manutenção do LTQ, ArcelorMittal Tubarão, Vitória, ES, Brasil.

\footnotetext{
* Contribuição técnica ao 69 Congresso Anual da ABM - Internacional e ao 14ํㅡㄹ ENEMET - Encontro Nacional de Estudantes de Engenharia Metalúrgica, de Materiais e de Minas, 21 a 25 de julho de 2014, São Paulo, SP, Brasil.
} 


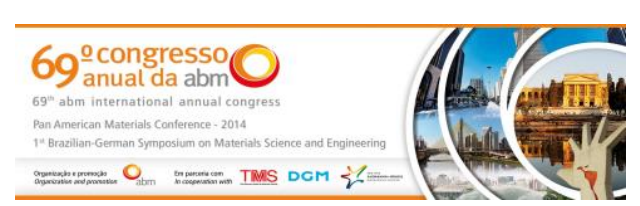

esboço é bobinado no Coil Box para homogeneizar a temperatura e inverter a ponta mais fria com a ponta mais quente. A tesoura de pontas corta as extremidades do esboço e direciona para o trem acabador composto por 6 cadeiras de laminação contínua. Ao sair do trem acabador a tira, com espessura entre 0,8 a $16 \mathrm{~mm}$, é conduzida à mesa de rolos onde estão instalados os chuveiros de resfriamento que conferem propriedades mecânicas e metalúrgicas a tira. As bobinadeiras, situadas no fim da linha de laminação, processam o bobinamento da tira em sua forma final, bobinas a quente. O produto final é identificado através de um código alfanumérico pela Máquina de Marcar, foco do estudo, antes de seguir para o pátio de estocagem e despacho.

\subsection{Sistema de Marcação de Bobinas}

A Máquina de Marcar Bobinas a quente realiza a identificação alfanumérica de todas as bobinas produzidas pelo Laminador de Tiras a Quente da ArcelorMittal Tubarão. A capacidade instalada é de 4 milhões de toneladas por ano.

A atividade de marcação é totalmente automatizada e robotizada podendo ser configurada para marcação na lateral da bobina ou na superfície externa (circunferência externa). Antes do robô iniciar a marcação ele detecta o raio da bobina através de um cilindro pneumático, retorna para a posição de marcação e a inicia. O comando e sequenciamento é realizado automaticamente pelo sistema de nível 2.

$\mathrm{Na}$ ocorrência de falha neste sistema de marcação, o operador imediatamente inicia a marcação em manual. Como as bobinas estão em torno de $800^{\circ} \mathrm{C}$ esta operação é bastante desconfortável e não possui o mesmo rendimento da marcação automatizada, ou seja, após poucos minutos de falha é necessário reduzir o ritmo do Laminador de Tiras a Quente em função da marcação manual. Normalmente esta operação reduz $50 \%$ da capacidade de produção do laminador. As bobinas a serem marcadas se acumulam gerando a parada completa do Laminador.

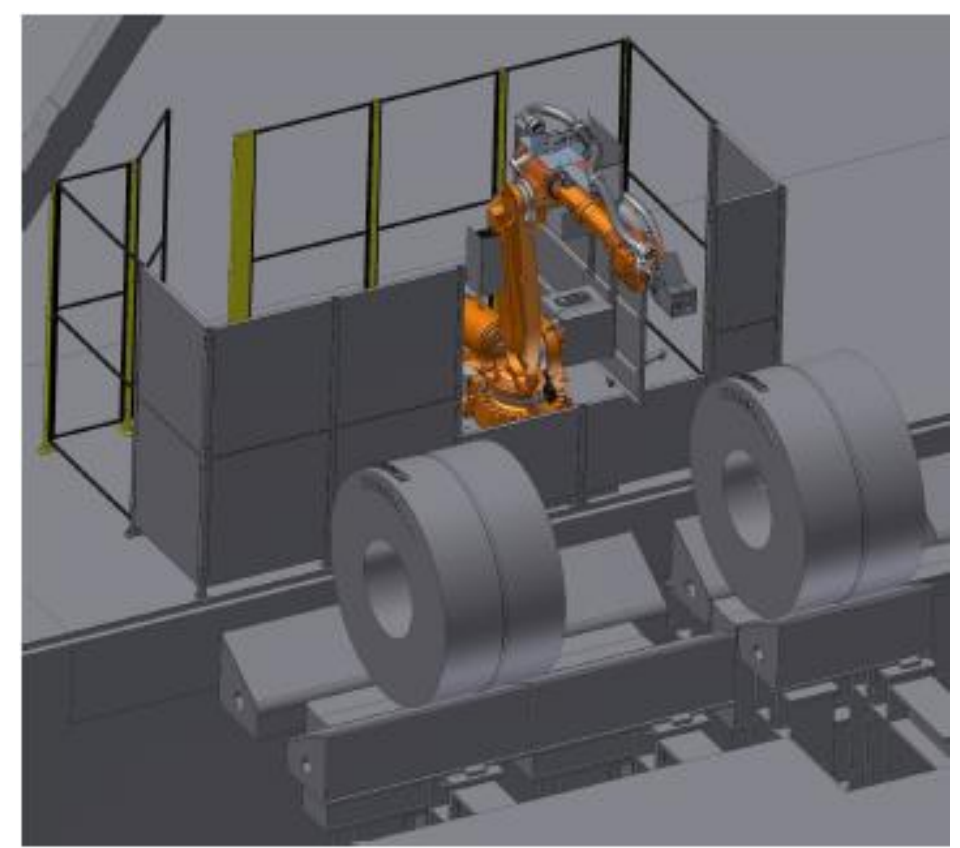

Figura 2. Vista ilustrativa da Máquina de Marcar Bobinas do Laminador de Tiras a Quente da ArcelorMittal Tubarão.

* Contribuição técnica ao 69 Congresso Anual da ABM - Internacional e ao 14ํㅡㄹ ENEMET - Encontro Nacional de Estudantes de Engenharia Metalúrgica, de Materiais e de Minas, 21 a 25 de julho de 2014, São Paulo, SP, Brasil. 


\section{MATERIAIS E MÉTODOS}

\subsection{Diagrama de Blocos Funcionais}

O primeiro passo do trabalho foi desenvolver o diagrama de blocos funcionais (FBD) do sistema de marcação de bobinas com o intuito de melhor entender o sistema e ter certeza que nenhuma parte e/ou componente ficaria fora da análise. O FBD foi desenvolvido suportado por visitas de campo e consulta a desenhos técnicos e manuais.

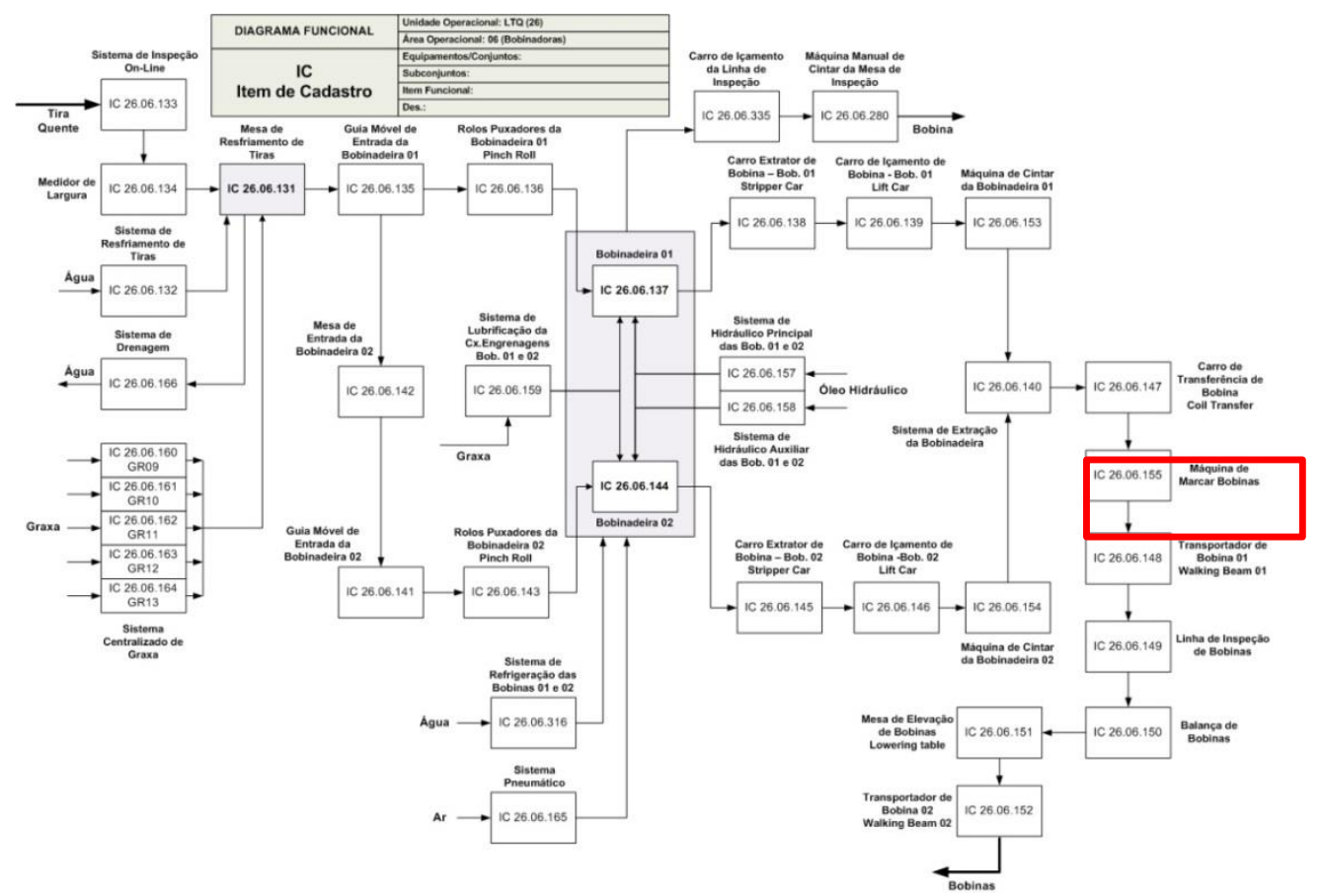

Figura 3. Diagrama de blocos funcionais da região da Mesa de resfriamento e Bobinadeiras do Laminador de Tiras a Quente da ArcelorMittal Tubarão.

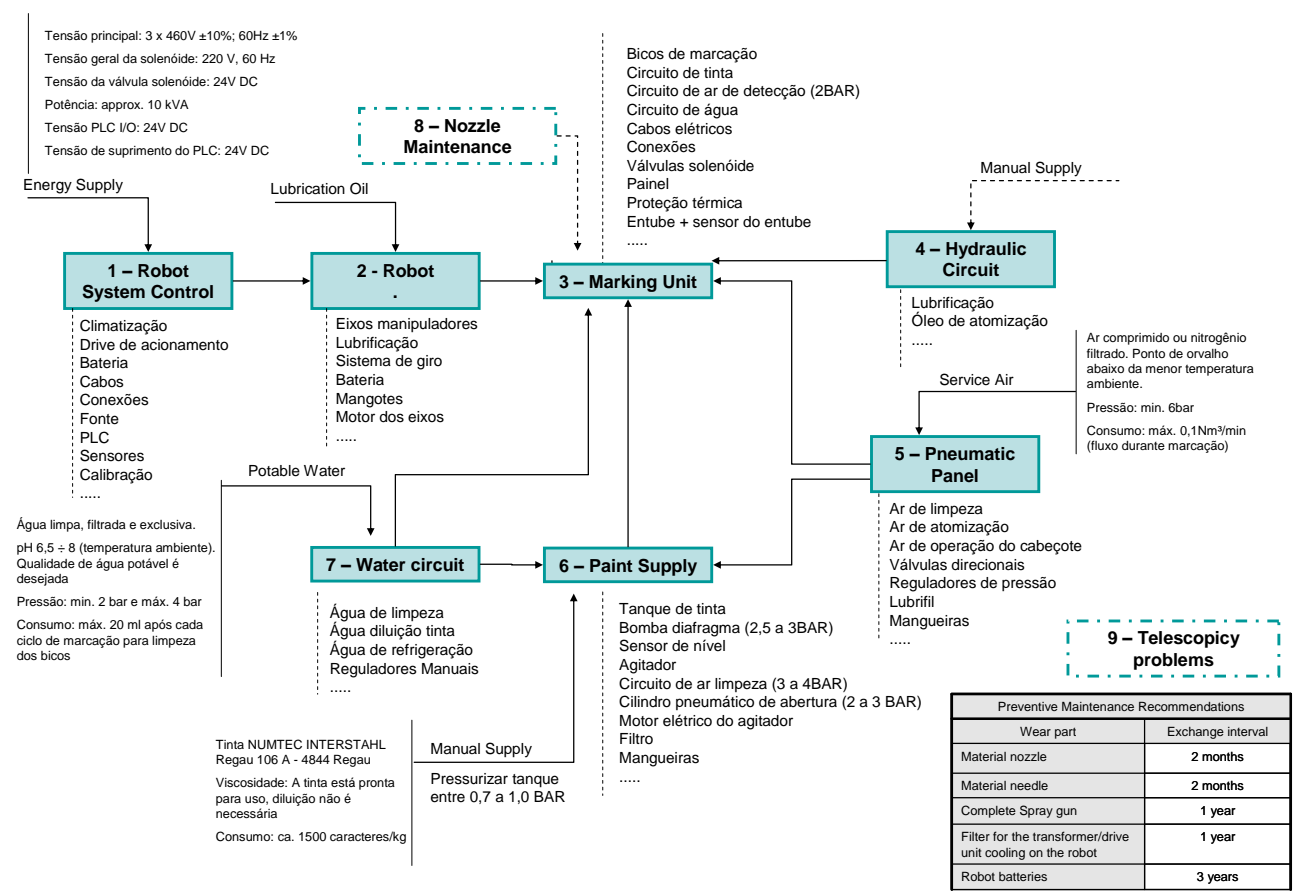

Figura 4. Diagrama de blocos funcionais da Máquina de Marcar do Laminador de Tiras a Quente da ArcelorMittal Tubarão.

* Contribuição técnica ao $69^{\circ}$ Congresso Anual da ABM - Internacional e ao 14ํㅡㄹ ENEMET - Encontro Nacional de Estudantes de Engenharia Metalúrgica, de Materiais e de Minas, 21 a 25 de julho de 2014, São Paulo, SP, Brasil. 


\subsection{Modelos Matemáticos}

Todas as falhas da máquina de marcar foram estratificadas e alocadas em cada bloco funcional. Logo foi possível desenvolver um modelo matemático para representar a probabilidade de falha do componente e outro para representar a probabilidade de reparo do componente. Este processo foi realizado para todos os blocos funcionais definidos no FBD. Para isto foi utilizado o programa Weibull ++, onde foi possível definir a melhor distribuição estatística a ser utilizada para cada caso [8].

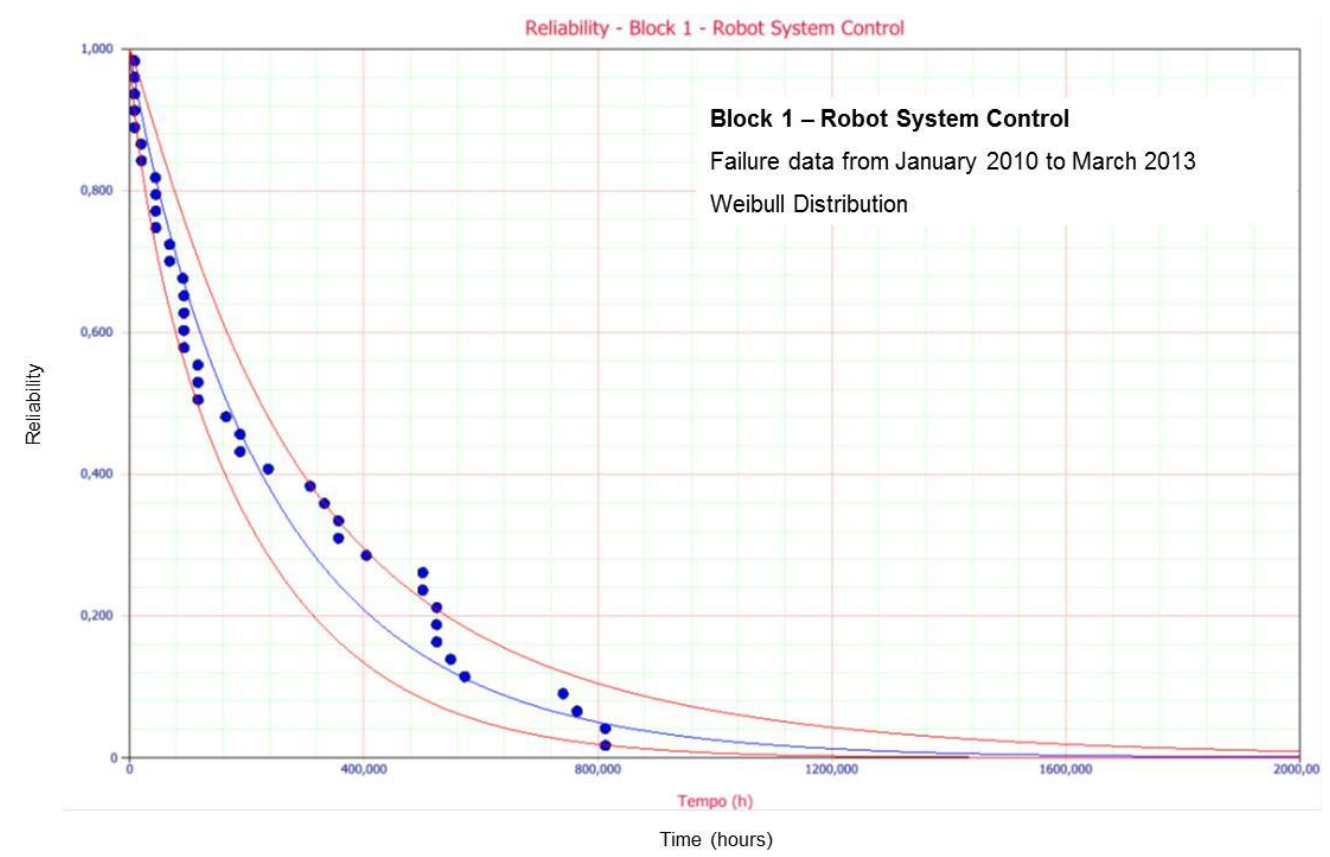

Figura 7. Exemplo de curva de confiabilidade gerada para um bloco funcional da Máquina de Marcar.

\subsection{Diagrama de Blocos de Confiabilidade}

A máquina de marcar e seus componentes foram representados através de diagrama de blocos de confiabilidade (RBD). Para cada equipamento representado pelo bloco foram incluídos os modelos matemáticos representando a frequência de falha e os tempos de reparo obtidos internamente nos registros históricos. Foi utilizado o programa Blocksim para tal [9]. As primeiras análises de simulação dos dados de falhas da Máquina de marcar indicaram um $\mathrm{R}$ (24horas) $=43,28 \%$ e um $\mathrm{R}(168 \mathrm{horas})=3,74 \%$, ou seja, a máquina de marcar tem uma probabilidade de $43,28 \%$ de não falhar em 24 horas e de $3,74 \%$ de não falhar em uma semana (168horas). Estes valores foram totalmente condizentes com o dia a dia da manutenção da área.

\footnotetext{
* Contribuição técnica ao 69ํ Congresso Anual da ABM - Internacional e ao 14ํㅡㄹ ENEMET - Encontro Nacional de Estudantes de Engenharia Metalúrgica, de Materiais e de Minas, 21 a 25 de julho de 2014, São Paulo, SP, Brasil.
} 


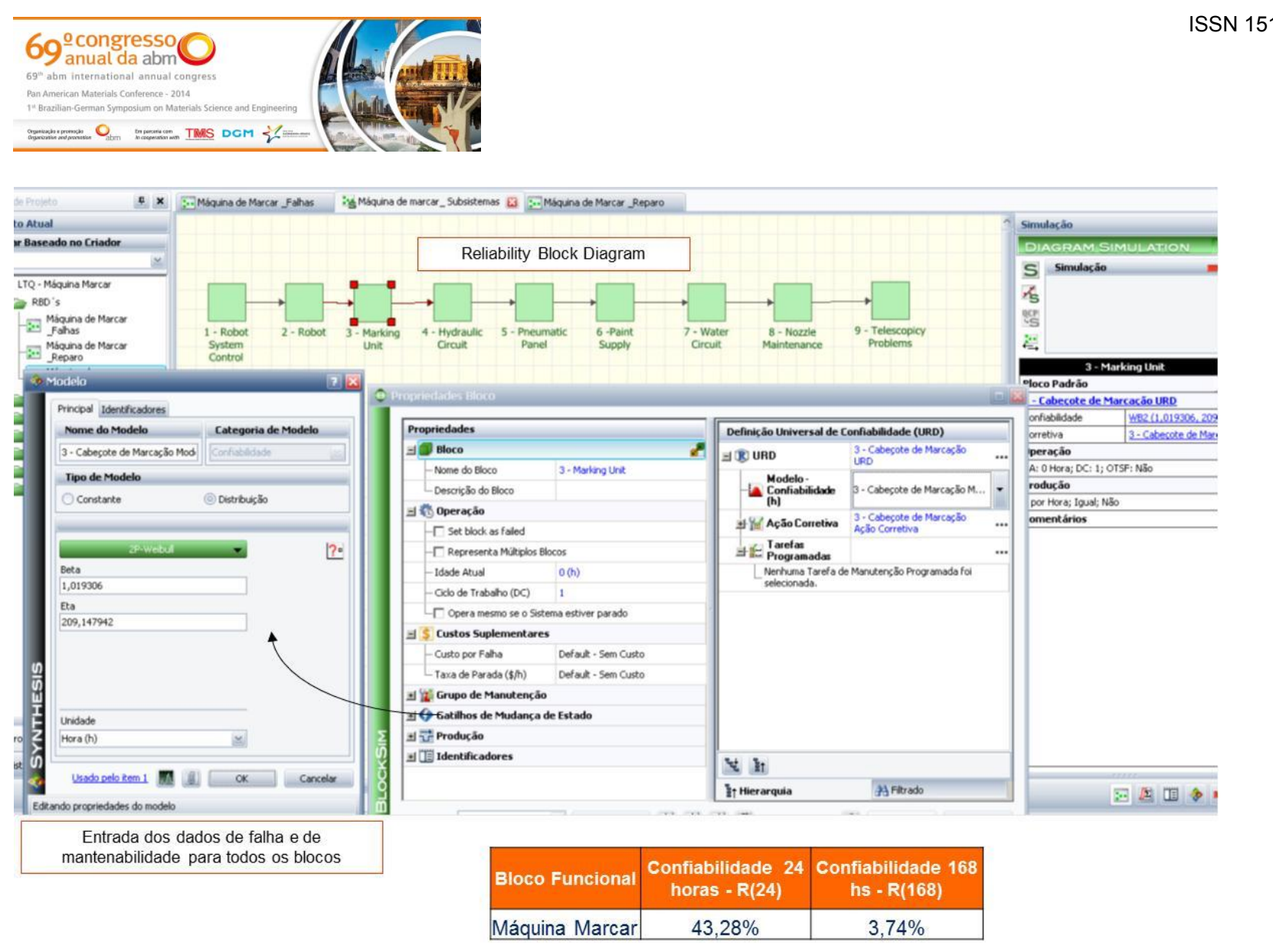

Figura 8. Primeiros resultados da simulação do diagrama de blocos de confiabilidade da Máquina de Marcar.

\section{RESULTADOS E DISCUSSÃO}

As análises de simulação realizadas no programa Blocksim mostram os subsistemas da máquina de marcar que mais contribuem para a baixa confiabilidade do sistema. De acordo com estes resultados o foco foi direcionado aos blocos:

- Sistema de controle do robô;

- Robô próprio;

- Unidade de Marcação;

- Manutenção de Bicos.

Tabela 1. Resultados das simulações do diagrama de blocos de confiabilidade

\begin{tabular}{l|c|c|c|}
\hline \multicolumn{1}{|c|}{ Diagrama Funcional } & $\begin{array}{c}\boldsymbol{\mu} \text { (MTTR) - } \\
\text { horas }\end{array}$ & $\begin{array}{c}\text { Confiabilidade } \\
\mathbf{2 4} \text { horas - R(24 }\end{array}$ & $\begin{array}{c}\text { Confiabilidade } \\
\mathbf{1 6 8} \text { hs - R(168) }\end{array}$ \\
\hline 1 - Sistema de Controle do Robô & 0,24 & $89,30 \%$ & $49,90 \%$ \\
\hline 2 - Robô & 0,62 & $79,90 \%$ & $60,70 \%$ \\
\hline 3 - Unidade de Marcação & 0,18 & $89,60 \%$ & $44,90 \%$ \\
\hline 4 - Unidade Hidráulica & 0 & $100 \%$ & $100 \%$ \\
\hline 5 - Painel Pneumático & 0,19 & $95,90 \%$ & $88,40 \%$ \\
\hline 6 - Suprimento de Tinta & 0,27 & $94,20 \%$ & $92,70 \%$ \\
\hline 7 - Circuito de Água & $6,96^{*}$ & $93,00 \%$ & $84,00 \%$ \\
\hline 8 - Manutenção dos Bicos & 0,28 & $87,60 \%$ & $51,90 \%$ \\
\hline 9 - Telescopicidade & 0,13 & $92,00 \%$ & $77,10 \%$ \\
\hline *Não representativo, pois ocorreram apenas duas ocorrências no período analisado.
\end{tabular}

Para cada um dos 4 blocos destacados foram realizados o detalhamento dos modos de falha e suas causas principais de forma a possibilitar a criação de plano de ação

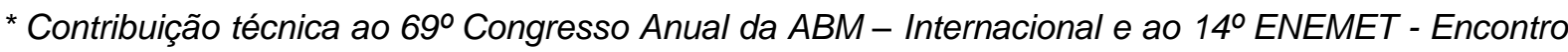
Nacional de Estudantes de Engenharia Metalúrgica, de Materiais e de Minas, 21 a 25 de julho de 2014, São Paulo, SP, Brasil.
} 
enxuto e focado no aumento de disponibilidade do sistema máquina de marcar $[7,10]$. Foram geradas nove ações relacionadas aos blocos destacados e mais 5 ações gerais para sustentabilidade do resultado. A implantação das ações foi monitorada semanalmente através de reuniões de follow up com os responsáveis e gerentes envolvidos. A gestão das ações de forma eficiente e motivacional para o grupo é fator fundamental para a implantação de qualquer ação.

Tabela 2. Plano de Ação focado nos blocos funcionais destacados

\begin{tabular}{|c|c|c|c|}
\hline BLOCO & AÇÔES & RESPONSÁVEL & DATA \\
\hline \multirow[t]{2}{*}{3} & $\begin{array}{l}1 \text { - Trocar a unidade de marcação existente ( } 7 \text { bicos) para marcação simples (um } \\
\text { bico). }\end{array}$ & Quintino & $03 / 07 / 2013$ \\
\hline & 2 -Instalação de filtro individualizado para o circuito pneumático. & Quintino/ Denilson & $03 / 07 / 2013$ \\
\hline \multirow{2}{*}{1} & 3 - Isolar termicamente a unidade de marcação & Quintino/ Denilson & $03 / 07 / 2013$ \\
\hline & 4-Melhorar a ventilação do local - Instalar ventilador industrial & Denilson & $31 / 10 / 2013$ \\
\hline \multirow{3}{*}{8} & $\begin{array}{l}5 \text { - Transferir a manutenção dos bicos de marcação para a Oficina Central para } \\
\text { melhorar o controle de qualidade }\end{array}$ & Sodré / Laudelino & $30 / 06 / 2013$ \\
\hline & 6 - Treinar os inspetores e executantes direto com o fabricante Numtec & $\begin{array}{l}\text { Quintino / } \\
\text { Fracalossi }\end{array}$ & $\begin{array}{c}25 / 06 \text { a } \\
02 / 07 / 2013\end{array}$ \\
\hline & $\begin{array}{l}7 \text { - Criar infraestrutura de reparo e teste dos bicos de marcação com água na } \\
\text { Oficina central }\end{array}$ & $\begin{array}{l}\text { Sodré / Laudelino / } \\
\text { José Anilton }\end{array}$ & $16 / 08 / 2013$ \\
\hline \multirow[b]{2}{*}{2} & 8 - Criar padrão visual dos códigos de falha e soluções rápidas & Denilson & $31 / 07 / 2013$ \\
\hline & $\begin{array}{l}9 \text { - Treinar a manutenção elétrica de turno na análise e diagnose de falhas do } \\
\text { robô, baseado no padrão criado. }\end{array}$ & Denilson & $30 / 08 / 2013$ \\
\hline \multirow{5}{*}{ ALL } & $\begin{array}{l}10 \text { - Criar rotina de análise aprofundada de todas as falhas da máquina de marcar } \\
\text { acima de } 60 \text { minutos. }\end{array}$ & $\begin{array}{l}\text { Luis Eduardo } \\
\text { Fontes }\end{array}$ & $12 / 07 / 2013$ \\
\hline & 11 - Revisar o cadastro do equipamento no CMMS. & $\begin{array}{l}\text { Sodré / Laudelino / } \\
\text { Quintino / Pianca }\end{array}$ & $30 / 06 / 2013$ \\
\hline & $\begin{array}{l}12 \text { - Revisar os planos de manutenção e inspeção baseado em RCM (Reliability } \\
\text { Centered Maintenance). }\end{array}$ & $\begin{array}{l}\text { Sodré / Laudelino / } \\
\text { Quintino / Pianca }\end{array}$ & $30 / 08 / 2013$ \\
\hline & $\begin{array}{l}13 \text { - Revisar o plano de inspeção incluindo ajuste semanal das pressões da } \\
\text { máquina de marcar. }\end{array}$ & Denilson & $31 / 07 / 2013$ \\
\hline & $\begin{array}{l}14 \text { - Instalar novo sistema de uniformização e suprimento de tinta para evitar o } \\
\text { contato do operador }\end{array}$ & Denilson / Quintino & $31 / 10 / 2013$ \\
\hline
\end{tabular}

Após a implantação das ações foi possível apurar ganhos com a redução do tempo de paradas do Laminador de Tiras a Quente causados pela Máquina de Marcar e também uma redução da perda de produtividade causada pela marcação manual. Podemos observar que foi possível atingir 0,77horas de parada do Laminador no mês de novembro contra uma média mensal de 4,49horas em 2013H1, e 0(zero) horas de perda de produtividade no mês de novembro contra 1,4horas de média mensal em 2013H1.

\footnotetext{
* Contribuição técnica ao 69 Congresso Anual da ABM - Internacional e ao 14ํㅡㄹ ENEMET - Encontro Nacional de Estudantes de Engenharia Metalúrgica, de Materiais e de Minas, 21 a 25 de julho de 2014, São Paulo, SP, Brasil.
} 


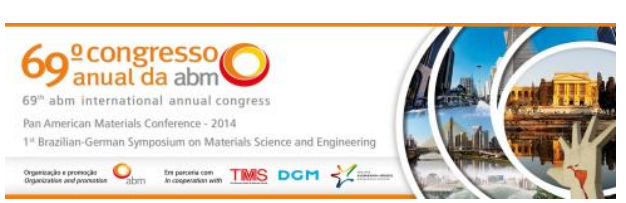

\section{Stop - Tempo de parada do LTQ causado por falha da Máquina de Marcar}

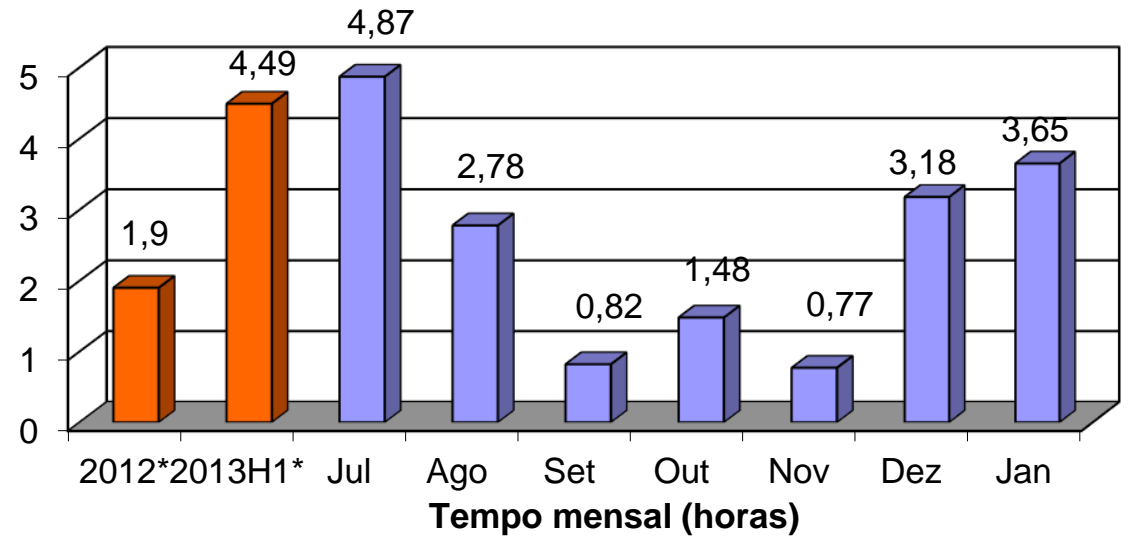

${ }^{*}$ Para 2012 e 2013H1 o valor representa a média mensal.

Figura 9. Resultados para o tempo de parada do Laminador causado pela Máquina de Marcar.

\section{Delay - Tempo de perda de produtividade do LTQ causado por falha da Máquina de Marcar}

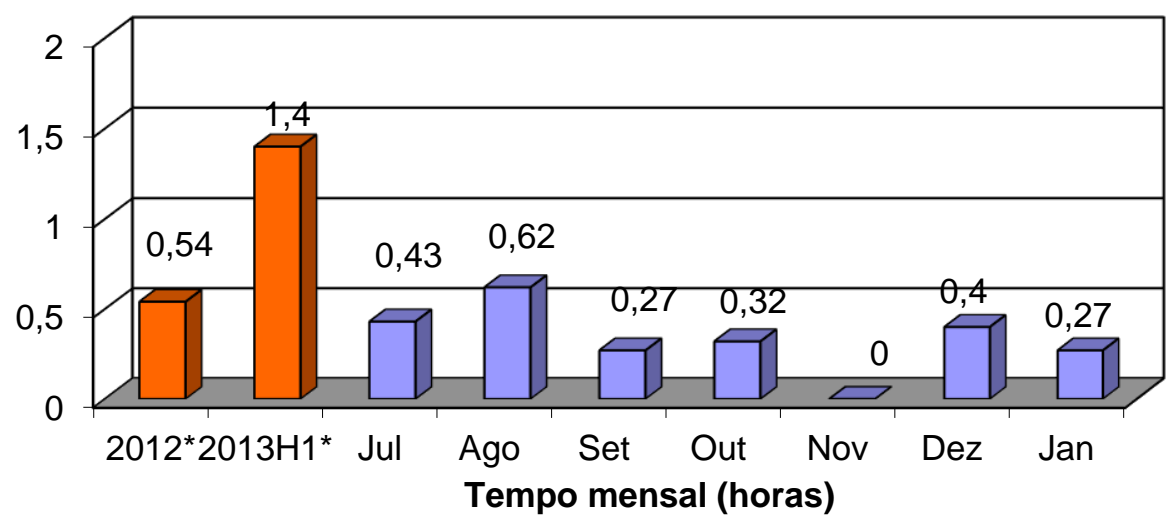

${ }^{*}$ Para 2012 e 2013H1 o valor representa a média mensal.

Figura 10. Resultados para o tempo de perda de produtividade do Laminador causado pela Máquina de Marcar.

Podemos observar que em dezembro de 2013 se inicia um aumento dos níveis de falhas, ou seja, as ações implantadas não foram suficientes para garantir a sustentabilidade do resultado. Então se decidiu realizar a revisão dos planos de manutenção e inspeção baseados no RCM (Manutenção Centrada em Confiabilidade) $[10,11,7]$.

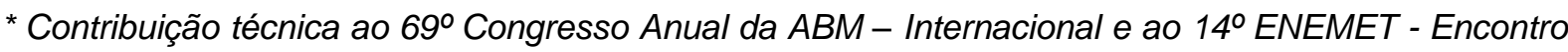
Nacional de Estudantes de Engenharia Metalúrgica, de Materiais e de Minas, 21 a 25 de julho de 2014, São Paulo, SP, Brasil.
} 

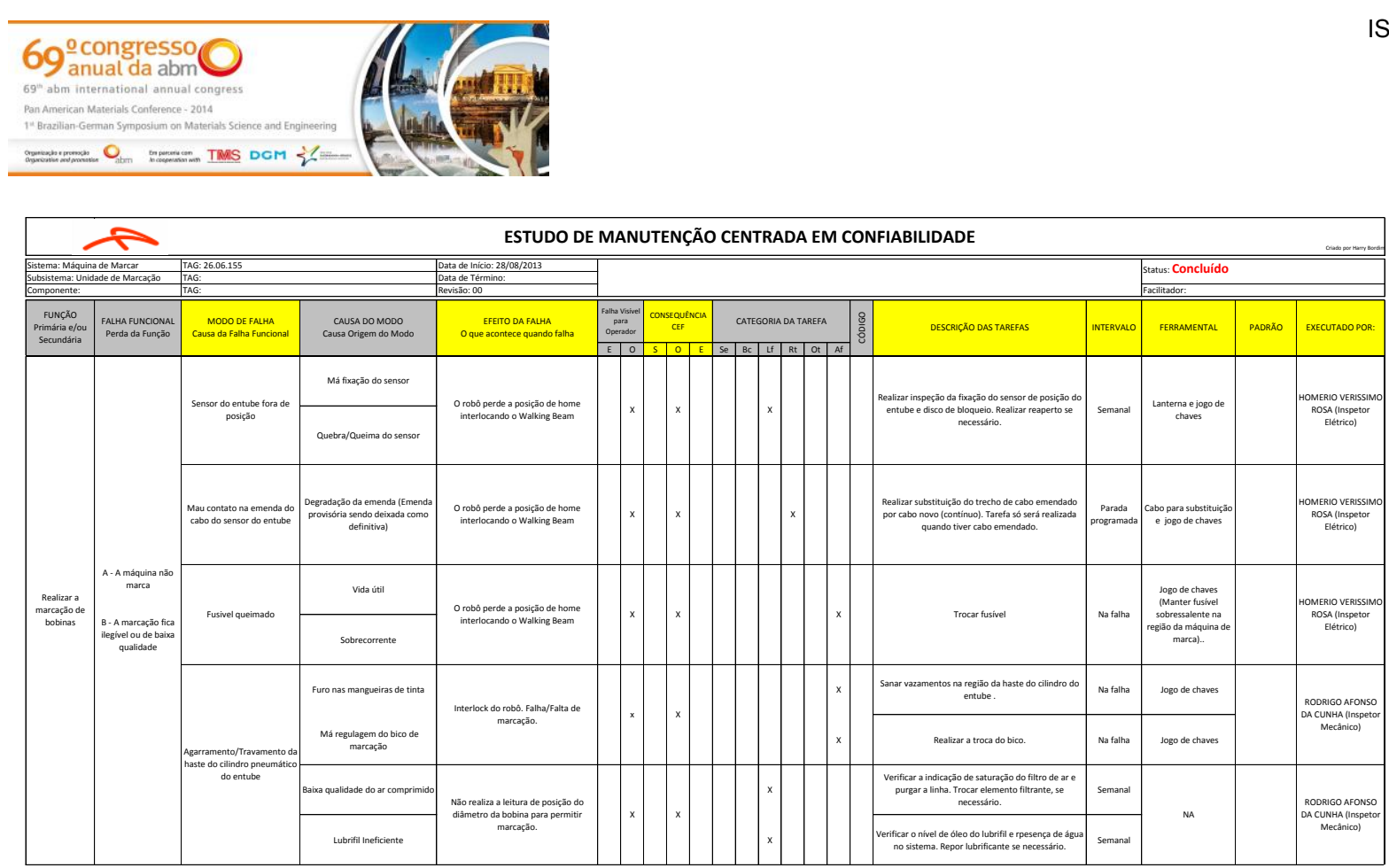

Figura 10. Exemplo de planilha de RCM utilizada na revisão dos planos de manutenção e inspeção.

Como resultado do estudo de 9 planos de serviços existentes 8 foram cancelados e 3 novos incluídos. Dos 61 planos de inspeção existentes, 40 foram cancelados e 22 novos foram incluídos. Foram incluídas 11 novas recomendações nos padrões de manutenção. Esta ação será fundamental para garantir a sustentabilidade do resultado.

\section{CONCLUSÕES}

A Engenharia de Confiabilidade Quantitativa, aplicada de forma estruturada, permitiu a realização da análise aprofundada dos equipamentos/componentes e modos de falhas da máquina de marcar. O principal benefício da aplicação estruturada foi 0 direcionamento do plano de ação em atividades que realmente iriam trazer resultados palpáveis, tornando o plano de ação enxuto e eficaz.

A redução do tempo de parada por falha da máquina de marcar proporcionou um aumento 14,26horas de disponibilidade do Laminador de Tiras a Quente e um aumento de 6,52 horas de produtividade total do Laminador. Considerando a produtividade média do Laminador, esse ganho se transforma em aproximadamente 15.207 ton/ano a mais de produção de bobinas a quente, que é o produto da ArcelorMittal Tubarão de maior valor agregado.

Pode-se observar que a garantia da sustentabilidade dos resultados deve ser realizada através de ações de revisão de padrões, planos de manutenção, inspeção e treinamento constante das pessoas.

\section{REFERÊNCIAS}

1 Xavier JN. Gestão para Manutenção Classe Mundial. Belo Horizonte: Tecem tecnologia Empresarial; 2005.

2 Kardec A, Xavier JN. Manutenção - Função Estratégica. 4ª edição. São Paulo: Qualitymark; 2013.

3 Associação Brasileira de Normas Técnicas. NBR 5462: Confiabilidade e Mantenabilidade. Rio de Janeiro; 1994.

4 Reliasoft Corporation. Análise de dados de vida. Presentation Handouts; 2012.

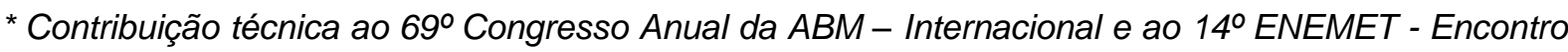
Nacional de Estudantes de Engenharia Metalúrgica, de Materiais e de Minas, 21 a 25 de julho de 2014, São Paulo, SP, Brasil.
} 
5 Seixas ES. Engenharia de Confiabilidade Aplicada na Manutenção [apostila da Abraman - RFFSA]. Belo Horizonte:Abraman; 1999

6 Reliasoft Corporation. Confiabilidade de Sistemas Avançados. Presentation Handouts; 2012.

7 NASA, Reliability centered maintenance guide for facilities and collateral equipment. Estados Unidos: NASA; 2000.

8 ReliaSoft Corporation, Weibull++ 8.0 Software Package, Tucson, AZ, Disponível em: $<$ http://www.Weibull.com>.

9 Tucson AZ. Blocksim 8.0 Software Package. ReliaSoft Corporation. Disponível em: $<$ http://www.ReliaSoft.com>.

10 Helman $\mathrm{H}$, Andery PRP. Análise de falhas (aplicação dos métodos de FMEA - FTA)" Série Ferramentas da qualidade. volume 11. Belo Horizonte: Fundação Cristiano Otoni, Universidade Federal de Minas Gerais; 1995.

11 MIL-STD-1629A - Procedures for performing a failure mode, efects and criticality analysis. Department of Defense - United States of America; 1980.

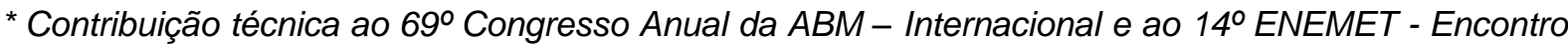
Nacional de Estudantes de Engenharia Metalúrgica, de Materiais e de Minas, 21 a 25 de julho de 2014, São Paulo, SP, Brasil. 